Revista de

Contabilidade e

Organizações

www.rco.usp.br
DOI: http://dx.doi.org/10.11606/rco.v10i26.102686
Journal of

Accounting and

Organizations

www.rco.usp.br

\title{
Análise da intensidade de adoção de indicadores de desempenho: uma abordagem contextual a partir de uma perspectiva institucional
}

\author{
Antonio André Cunha Callado ${ }^{\mathrm{a}}$, Rezilda Rodrigues Oliveira ${ }^{\mathrm{a}}$
}

${ }^{a}$ Universidade Federal Rural de Pernambuco

\section{Informações do Artigo}

Histórico do Artigo

Recebido: 23 de agosto de 2015

Aceito: 24 de abril de 2016

Palavras-chave:

Indicadores de desempenho.

Características das empresas.

Teoria Institucional.

\begin{abstract}
Resumo
Empresas podem possuir características técnicas e desempenhar diferentes funções operacionais, bem como possuir culturas organizacionais, crenças e valores distintos. O objetivo deste artigo é identificar a presença de especificidades acerca da intensidade de uso acerca de indicadores de desempenho a partir de uma perspectiva institucional advinda de distintas funções operacionais executadas por empresas no âmbito de uma cadeia agroalimentar. Foi operacionalizado um survey junto a 121 empresas que foram agrupadas de acordo com suas respectivas funções operacionais dentro da cadeia. Os dados relativos ao uso/não uso de 32 indicadores de desempenho foram coletados através de um questionário apresentado ao gestor principal de cada uma das empresas investigadas. A análise dos resultados considerou a Prova Exata de Fisher e a Análise de Agrupamentos. Foi revelado que a intensidade de uso da maioria dos indicadores de desempenho difere entre os grupos de empresas analisados, bem como que grupos específicos de indicadores de desempenho foram identificados para cada uma das funções operacionais investigadas.
\end{abstract}

\section{INTRODUÇÃO}

Cadeia de suprimento pode ser definida como uma rede de três ou mais empresas através das quais os produtos fluem a partir de fontes primárias para os consumidores finais (MIN; MENTZER; LADD, 2007; ELROD; MURRAY; BANDE, 2013). Em se tratando da Teoria Institucional, sabe-se que esta apresenta duas modalidades distintas de mecanismos de coordenação de cadeias de suprimento, pelas quais as relações comerciais realizadas ao longo de sua estrutura se baseiam: na hierarquia, no caso de cadeias integradas; ou em operações de mercado, para as cadeias não integradas (WILLIAMSON, 1975). O mecanismo de hierarquia deve ser considerado apenas se o mercado não pode fornecer proteção eficiente contra o oportunismo (HANN et al, 2003).

Empresas que atuam em diferentes estágios de uma cadeia de suprimentos são interdependentes e influenciam seus desempenhos (HAAN et al., 2003; BIGLIARDI; BOTTANI, 2010). Quando uma cadeia é composta por empresas integradas se comportando como uma entidade única, a mensuração de seu desempenho deve abranger toda sua estrutura (HOLMBERG, 2000; BIGLIARDI; BOTTANI, 2010) e o escopo dos indicadores de desempenho deve considerar a cadeia como um todo (VAN HOEK, 2001). Por outro lado, quando uma cadeia é composta por empresas não integradas que desempenham distintas funções, os indicadores de desempenho devem estar associados a seus respectivos focos gerenciais (VAN HOEK, 1998; LAMBERT; POHLEN, 2001).

Cadeia agroalimentar de suprimentos é uma rede de empresas que trabalham juntos para fornecer produtos agrícolas aos consumidores finais (CHIRSTOPHER, 2005). No entanto, Van der Vorst (2006) afirma que no âmbito desta modalidade de cadeias de suprimento há uma maior tendência de que empresas que as compõem mantenham sua autonomia em comparação às demais modalidades.

A estrutura de uma cadeia agroalimentar de suprimentos pode ser complexa, composta por inúmeras entidades individuais, bem como possuir numerosas interações entre elas (MATOPOULOS; VLACHOPOULOU;

Autor Correspondente: Tel (81) 3320-5403

E-mail: andrecallado@yahoo.com.br (A. A. C. Callado); rezilda.rodrigues@gmail.com (R. R. Oliveira)

UFRPE - Rua D. Manoel de Medeiros, s/s. Dois Irmãos - Recife-PE, CEP: 52.171-900, Brasil. 
MANTHOU, 2004). Diversas funções operacionais específicas, tais como fornecedores de insumos, produtores rurais, beneficiadores, distribuidores e varejistas podem ser identificadas ao longo da estrutura de uma cadeia agroalimentar de suprimentos (HENSON; REARDON, 2005).

A abordagem institucional dos estudos organizacionais tem se firmado como uma referência teórica relevante sobre as discussões inerentes aos ambientes institucionais, bem como seus efeitos sobre as estruturas e processos organizacionais. $\mathrm{O}$ ambiente institucional representa um enriquecimento do que se compreende como ambiente técnico, ampliado ao domínio do simbólico (CARVALHO; VIEIRA; LOPES, 1999). Com efeito, uma instituição surge pela repetição de um hábito da atividade humana e a sua institucionalização se dá quando há uma tipificação recíproca de ações habituais por determinados tipos de atores (SELZNICK, 1996). O entendimento é de que esses scripts infundem valor ao processo de institucionalização, cujo estudo equivale a se ter compreensão acerca de como estruturas, incluindo esquemas, regras, normas e rotinas não apenas emergem como também são difundidos, adotados e adaptados ao longo do espaço e do tempo, assim como passam por declínio e desuso (TOLBERT; ZUCKER, 1998).

A partir do entendimento institucional decorrente da tipificação recíproca de ações habituais por determinados tipos de atores organizacionais, parece plausível que se possa capturar a forma pela qual o comportamento organizacional pode ser moldado na medida em que regras, normas e crenças compartilhadas produzem impactos no funcionamento da organização e influenciam a determinação dos requisitos necessários ao seu bom desempenho, criando as lentes através das quais elas veem o mundo, selecionam formas estruturais, operacionalizam sua ação e enfrentam as pressões ambientais (DiMAGGIO; POWELL, 1991). Meyer, Scott e Deal (1992) supõem que as organizações buscam adquirir domínio sobre o ambiente justamente para obter sucesso no uso das regras e especificações (conformidades) que induzem sua forma de gerenciamento.

Práticas gerenciais têm sido entendidas como associadas à natureza das características operacionais inerentes ao setor de atividade econômica do qual as empresas fazem parte (CALLADO, PINHO, 2014). Além disso, a contextualização do processo gerencial das diversas atividades econômicas é uma preocupação contínua (KAPLAN; COOPER, 1998). Além disso, características inerentes às empresas, tais como, políticas gerenciais e rotinas operacionais podem exercem influência sobre a maneira pela qual a importância acerca dos indicadores de desempenho pode ser percebida (HOLMBERG, 2000; PARK; LEE; YOO, 2005).

A definição dos indicadores apropriados para medir o desempenho de cadeias de suprimento entre distintas empresas não é uma tarefa fácil e, como já mencionado, há pouca literatura que faça referência a métodos específicos voltados para sua seleção (CHAN et al, 2003). Contudo, abordagens específicas destinadas a identificar indicadores comuns adotados por empresas que atuam ao longo de uma cadeia pode fornecer uma contribuição significativa para este campo de estudo (LAMBERT; POHLEN, 2001).

O objetivo deste artigo é identificar a presença de especificidades acerca da adoção de indicadores de desempenho a partir de uma abordagem institucional advinda de distintas funções operacionais executadas por empresas individuais no âmbito de uma cadeia agroalimentar.

Este trabalho possui a seguinte estrutura. Inicialmente, são apresentadas as considerações introdutórias e o objetivo da pesquisa. Em seguida são descritos os principais aspectos conceituais referentes à mensuração de desempenho no contexto de cadeias de suprimento. Posteriormente, são descritos os procedimentos metodológicos adotados para a sua operacionalização. Finalmente, são apresentados os resultados e as conclusões.

\section{MENSURAÇÃO DE DESEMPENHO}

A medição do desempenho de cadeias de suprimento tem recebido bastante atenção dos acadêmicos e profissionais nas últimas décadas (GANGA; CARPINETTI, 2011), mas há muito pouca discussão na literatura disponível que lida com a seleção de indicadores de desempenho por parte de empresas não integradas no contexto da mensuração de desempenho de cadeias (BEAMON, 1998; BEAMON, 1999; CHAN; QI, 2003).

Apesar dos diversos aprimoramentos acerca dos métodos de medição de desempenho de cadeias de suprimento, a natureza do processo seleção de métricas relevantes ainda tem sido visto como fonte potencial de problemas (MELNYK; STEWART; SWINK, 2004). Pelo menos três questões relativas à investigação acadêmica acerca da mensuração do desempenho de cadeias de suprimento ainda requerem investigação mais aprofundada (BEAMON, 1998): a) Os sistemas de medição de desempenho existentes para avaliar cadeias de suprimento são 
adequados? b) Os indicadores de desempenho considerados são adequados para avaliar cadeias de suprimento? C) Há alguma hierarquia entre os indicadores diversos indicadores de desempenho?

Sistemas de medição de desempenho para cadeias de suprimento podem contribuir para a melhoria do processo de alinhamento estratégico de todas as empresas individuais que as compõem (FOLAN; BROWNE, 2005; CHENHALL, 2005; LEE; KWON; SEVERANCE, 2007). No entanto, vários fatores podem influenciar a contribuição que a medição de desempenho pode gerar para cadeias de suprimento, a saber (LAMBERT; POHLEN, 2001):

- A ausência de uma direção clara para a cadeia de suprimento;

- A complexidade da medição de desempenho entre as diversas empresas individuais ao longo de toda cadeia;

- O desejo de não compartilhar informações com outras empresas participantes;

- A dificuldade de captar o desempenho por parte dos clientes, produtos ou correntes específicos.

O conceito de cadeia de suprimento contém a noção de que exista algum tipo de interdependência entre as atividades realizadas pelas empresas que a compõem, independentemente da presença de hierarquia ou processo de coordenação de mercado entre eles (DUBOIS; HULTHÉN; PEDERSEN, 2004). O número de empresas que a compõe, a frequência de transações realizadas, as especificidades dos produtos e o nível de incerteza são os principais aspectos considerados para identificar o tipo mais adequado de coordenação para qualquer cadeia de suprimento (CABRAL, 2011). Neste sentido, o papel desempenhado pelas empresas dentro da estrutura operacional de uma cadeia de suprimento pode influenciar as suas decisões relativas à medição de desempenho (FOLAN; BROWNE, 2005).

Há um entendimento de que o desempenho de cadeia de suprimento possa ser abordado quando um indicador de desempenho escolhido seja adotado por todos as empresas integrantes, estabelecendo, assim, uma base comum para a avaliação da cadeia como um todo. A partir da identificação de indicadores utilizados por todas as empresas participantes, seu desempenho deve ser medido por esses indicadores (NEELY; GREGORY; PLATTS, 1995). Estudos de caso, Lohman, Fortuin e Woulters (2004), Park, Lee e Yoo (2005), Bhagwat e Sharm (2007), Varma, Wadhwa e Deshmukh (2008), Bigliardi e Bottani (2010) e Rajesh et al. (2012), apresentam abordagens para investigar a mensuração de desempenho da cadeias de suprimento. Chia et al. (2009) realizaram um estudo de levantamento, enquanto Chang (2009) realizou um estudo de caso seguido de uma pesquisa. Nestes estudos, os indicadores de desempenho foram classificados considerando as perspectivas do Balanced Scorecard.

Por outro lado, Morash (2002) e Kohlemainen (2010) entendem que a utilização de indicadores comuns para medir não deva ser considerada. Para estes autores, esta abordagem pode desconsiderar indicadores de desempenho relevantes e representativos envolvidos em cada etapa da cadeia de suprimentos em detrimento de indicadores comuns que possuem diferente importância relativa para cada uma das empresas participantes.

O desempenho de uma empresa pode ser avaliado, compreendido e ter valor estratégico através da perspectiva do seu papel como parte integrante da sequencia de operações que ocorre dentro de transações no mercado à vista do qual faz parte (CALLADO; CALLADO, 2011).

O desempenho de cadeias de suprimento deve considerar habilidades específicas e excelência operacional a partir de seus participantes (MORASH, 2002). Este ponto também é discutido por Kohlemainen (2010), que salienta a importância de situar a responsabilidade da escolha de indicadores de desempenho no nível da gestão das empresas dentro da cadeia.

Assim, a principal preocupação da medição de desempenho de cadeias de suprimento deve ser centrada na definição da lista de indicadores que realmente importam, considerando as especificidades das empresas participantes, de modo que o elenco de indicadores de desempenho propostos busque refletir com precisão os efeitos desejados e habilidades relevantes (HOFMAM, 2004). No entanto, merece ser destacado que os procedimentos referentes à seleção de indicadores de desempenho são, em certa medida, um tanto subjetivos (FOLAN; BROWNE, 2005) e incertos (LOHMAN; FORTUIN; WOULTERS, 2004).

Deve-se notar que a mensuração de desempenho no contexto das cadeias de suprimento é um campo de estudo ainda aberto à discussão, tanto devido às características das cadeias de suprimento, quanto a ampla gama de indicadores de desempenho disponíveis e as diferentes abordagens concebidas para cumprir esta tarefa. 


\section{PROCEDIMENTOS METODOLÓGICOS}

Considerando o objetivo proposto, um survey foi realizado entre empresas que atuam na cadeia agroalimentar de frutas frescas no Estado de Pernambuco. De acordo com a FAO (2013), o Brasil é o terceiro maior produtor de frutas do mundo, com aproximadamente 20 milhões de toneladas de frutas frescas produzidas em 1,1 milhões de hectares em todo o país, sendo que a União Europeia é o principal mercado do Brasil e consome mais de 70\% da produção nacional (BRAZILIAN FRUIT, 2014).

A estrutura operacional da cadeia de suprimentos de frutas frescas é composta por fornecedores de insumos, produtores, distribuidores e varejistas (MAPA, 2007). Esta cadeia é formada quase que exclusivamente por empresas individuais independentes que fazem negócios por meio de operações no mercado à vista, desde o fornecimento de insumos até a venda para os consumidores finais em busca de eficiência e flexibilidade (NEVES, 2003).

De acordo como o último Censo Agropecuário (IBGE, 2006), a população de empresas agroindustriais no Estado de Pernambuco é de aproximadamente 34.000 entidades. Considerando um nível de confiança de 95\% e um erro de 5\%, o tamanho da amostra deveria ser de aproximadamente 121 empresas. Considerando a impossibilidade de identificar todas as empresas do universo, empresas que atuam no agronegócio foram contactadas a partir da acessibilidade e convidadas a participar da pesquisa e fornecer as informações necessárias. Estas empresas foram agrupadas de acordo com suas funções específicas na cadeia de suprimento (31 fornecedores de insumos, 13 agricultores, 47 distribuidores e 30 varejistas). A tabela 1 mostra o perfil das amostras consideradas.

Tabela 1. Perfil das amostras (\%).

\begin{tabular}{|c|c|c|c|c|}
\hline Características & Fornecedores & Agricultores & Distribuidores & Varejistas \\
\hline \multicolumn{5}{|l|}{ Tamanho } \\
\hline Pequenas & 58,1 & 30,7 & 57,4 & 53,4 \\
\hline Médias & 12,8 & 15,4 & 14,9 & 23,3 \\
\hline Grandes & 29,1 & 53,9 & 27,7 & 23,3 \\
\hline \multicolumn{5}{|l|}{ Tempo de atuação no mercado } \\
\hline Até 10 anos & 29,1 & 15,3 & 36,1 & 46,6 \\
\hline Entre 11 e 20 anos & 22,5 & 38,5 & 38,3 & 43,3 \\
\hline Mais de 20 anos & 48,4 & 46,2 & 25,6 & 10,1 \\
\hline
\end{tabular}

Fonte: Pesquisa de campo.

Para esta pesquisa, foram considerados 32 indicadores de desempenho (CALLADO; MENDES; CALLADO, 2013). Para facilitar a visualização das finalidades gerenciais dos indicadores de desempenho escolhidos, eles foram classificados e distribuídos entre as quatro perspectivas do Balanced Scorecard da seguinte maneira:

- Indicadores financeiros: lucratividade; liquidez; faturamento por produto; faturamento por funcionário; margem de contribuição; endividamento; rentabilidade do patrimônio; custo unitário;

- Indicadores de clientes: satisfação dos clientes; fidelidade dos clientes; captação de novos clientes; participação no mercado; valor da marca; lucratividade por cliente; faturamento por cliente; eficiência dos revendedores e distribuidores;

- Indicadores de processos internos: novos produtos; novos processos; produtividade por unidade de negócio; devoluções; pós-venda; ciclo de produção; fornecedores; desperdício;

- Indicadores de aprendizado e crescimento: investimento em treinamento; investimento em tecnologia; investimento em sistemas de informação; motivação; capacidade dos funcionários; eficiência gerencial; satisfação dos funcionários.

Antes da coleta de dados, a fim de verificar a adequação do questionário, bem como para identificar a necessidade de novos ajustes, um pré-teste foi realizado junto a cinco empresas selecionadas especificamente para esta finalidade. Os procedimentos de coleta de dados foram semelhantes aos adotados por Chia, Goh e Hum 
(2009) e Lee, Kwon e Severance (2007) e todas as informações foram obtidas através de realização de entrevistas presenciais realizadas nas dependências das empresas participantes.

Os 32 indicadores de desempenho foram operacionalizados como variáveis binárias listados em um questionário e apresentados aos gestores seniores que foram solicitados a identificarem aqueles que têm sido usados por suas respectivas empresas. Foram obtidas as intensidades de uso dos indicadores de desempenho a partir dos seus respectivos percentuais de uso. Todas as entrevistas foram precedidas de contatos telefônicos através dos quais foram agendadas as datas. O critério utilizado para identificar os gerentes seniores foi a responsabilidade pela tomada de decisões estratégicas, independentemente do nome adotado para identificar o cargo ocupado. Os dados foram coletados entre 2013 e 2014 e todos os questionários foram respondidos integralmente.

Embora o tamanho de amostras individuais não tenha sido grande o suficiente para permitir o uso de testes estatísticos paramétricos, eles foram suficientes para criar uma base de dados (GUNASECARAN; PATEL; MCGAUGHEY, 2004). Para a análise dos resultados foram utilizadas duas técnicas estatísticas não paramétricas complementares.

A primeira técnica estatística não paramétrica foi a Prova Exata de Fisher. Este teste estatístico é apropriado quando a natureza dos dados não permite a utilização de outras técnicas estatísticas mais sofisticadas (LEVIN, 1987). O referido teste foi utilizado com a finalidade de analisar a significância estatística das diferenças entre o uso/não uso dos diversos indicadores de desempenho considerados a partir das funções operacionais das empresas na estrutura da cadeia para testar as seguintes hipóteses:

$\mathrm{H}_{0}$ : a intensidade de uso dos indicadores de desempenho é semelhante entre as funções operacionais desempenhadas pelas empresas.

$\mathrm{H}_{1}$ : a intensidade de uso dos indicadores de desempenho é diferente entre as funções operacionais desempenhadas pelas empresas.

Para realizar este teste, seis combinações de funções operacionais foram submetidas ao teste para testar as hipóteses, que foram:

- Fornecedores de insumos e agricultores;

- Fornecedores de insumos e distribuidores;

- Fornecedores de insumos e varejistas;

- Agricultores e distribuidores;

- Agricultores e varejistas;

- Distribuidores e varejistas.

Deste modo, valores de $\mathrm{p}$ inferiores ou iguais a 0,05 indicam a presença de diferenças estatisticamente significativas entre as intensidades de uso, rejeitando $\mathrm{H}_{0}$, enquanto valores superiores não a rejeitem.

A segunda técnica foi a análise multivariada de agrupamentos. Para Hair et al (2005), a análise multivariada é um conjunto de métodos estatísticos que simultaneamente analisam múltiplas medidas sobre cada um dos indivíduos ou objetos sob investigação e que a análise de relações de interdependência. Esta técnica foi utilizada para identificar a dinâmica de relações dos indicadores de desempenho que obtivessem até duas rejeições da hipótese nula testada através da Prova Exata de Fisher.

\section{RESULTADOS DA PESQUISA}

A partir da base de dados composta pelas respostas fornecidas pelos entrevistados, as técnicas estatísticas foram executadas em busca do objetivo proposto para esta pesquisa. Inicialmente foi utilizada a Prova Exata de Fisher considerando as seis combinações possíveis. Os resultados referentes aos indicadores de desempenho financeiros estão apresentados na tabela 2. 
Tabela 2. Resultados da prova Exata de Fisher acerca dos indicadores financeiros.

\begin{tabular}{lcccccc}
\hline \multicolumn{1}{c}{ Indicadores } & Fornecedor & Fornecedor & Fornecedor & Agricultor \\
testados & Agricultor & $\begin{array}{c}\mathbf{x} \\
\text { Distribuidor }\end{array}$ & $\begin{array}{c}\mathbf{x} \\
\text { Varejista }\end{array}$ & $\begin{array}{c}\mathbf{x} \\
\text { Distribuidor }\end{array}$ & $\begin{array}{c}\mathbf{x} \\
\text { Varejista }\end{array}$ & $\begin{array}{c}\text { Distribuidor } \\
\mathbf{x} \\
\text { Varejista }\end{array}$ \\
\hline Lucratividade & 0,46 & 0,01 & 0,11 & 0,17 & 0,08 & 0,00 \\
$\begin{array}{l}\text { Liquidez } \\
\begin{array}{l}\text { Faturamento por } \\
\text { produto }\end{array}\end{array}$ & 0,00 & 0,00 & 0,17 & 0,55 & 0,00 & 0,00 \\
$\begin{array}{l}\text { Faturamento por } \\
\text { empregado }\end{array}$ & 0,07 & 0,10 & 0,19 & 0,31 & 0,01 & 0,00 \\
$\begin{array}{l}\text { Margem de } \\
\text { contribuição }\end{array}$ & 0,02 & 0,06 & 0,50 & 0,44 & 0,02 & 0,01 \\
$\begin{array}{l}\text { Nível de } \\
\text { endividamento }\end{array}$ & 0,07 & 0,00 & 1 & 0,47 & 0,00 & 0,00 \\
$\begin{array}{l}\text { Rentabilidade do } \\
\text { patrimônio }\end{array}$ & 0,66 & 0,00 & 0,00 & 0,29 & 0,23 & 0,45 \\
\begin{tabular}{l} 
Custo unitário \\
\hline
\end{tabular} & 0,47 & 0,00 & 0,00 & 0,11 & 0,00 & 0,00 \\
\hline
\end{tabular}

Fonte: Pesquisa de campo.

Os resultados mostram que o indicador de desempenho referente à rentabilidade do patrimônio foi o único que não apresentou rejeição da hipótese nula entre as combinações de dois-por-dois. Tais resultados indicam que a intensidade de uso deste indicador de desempenho pode ser considerada como semelhante entre as empresas pesquisadas, independentemente das funções operacionais executadas pelas empresas. Os resultados dos outros indicadores de desempenho da perspectiva financeira mostram evidências (rejeições da hipótese nula), indicando a presença de diferenças significativas acerca da intensidade de uso entre as funções operacionais.

O mesmo teste foi realizado com os indicadores de desempenho associados aos clientes. Os resultados são apresentados na tabela 3 .

Tabela 3. Resultados da prova Exata de Fisher acerca dos indicadores associados aos clientes.

\begin{tabular}{|c|c|c|c|c|c|c|}
\hline $\begin{array}{l}\text { Indicadores } \\
\text { testados }\end{array}$ & $\begin{array}{c}\text { Fornecedor } \\
\qquad \mathbf{x} \\
\text { Agricultor } \\
\end{array}$ & $\begin{array}{l}\text { Fornecedor } \\
\mathbf{x} \\
\text { Distribuidor } \\
\end{array}$ & $\begin{array}{c}\text { Fornecedor } \\
\mathbf{x} \\
\text { Varejista } \\
\end{array}$ & $\begin{array}{c}\text { Agricultor } \\
\mathbf{x} \\
\text { Distribuidor } \\
\end{array}$ & $\begin{array}{c}\text { Agricultor } \\
\mathbf{x} \\
\text { Varejista } \\
\end{array}$ & $\begin{array}{c}\text { Distribuidor } \\
\mathbf{x} \\
\text { Varejista } \\
\end{array}$ \\
\hline $\begin{array}{l}\text { Satisfação dos } \\
\text { clientes }\end{array}$ & 0,58 & 0,10 & 0,23 & 0,30 & 0,44 & 0,44 \\
\hline $\begin{array}{l}\text { Fidelidade dos } \\
\text { clientes }\end{array}$ & 0,39 & 0,20 & 0,50 & 0,56 & 0,28 & 0,10 \\
\hline $\begin{array}{l}\text { Captação de } \\
\text { novos clientes }\end{array}$ & 0,16 & 0,00 & 0,00 & 0,14 & 0,22 & 0,49 \\
\hline $\begin{array}{l}\text { Participação de } \\
\text { Mercado }\end{array}$ & 0,07 & 0,25 & 0,00 & 0,18 & 0,00 & 0,00 \\
\hline Valor da marca & 0,02 & 0,41 & 0,01 & 0,00 & 0,00 & 0,02 \\
\hline $\begin{array}{l}\text { Lucratividade } \\
\text { por cliente }\end{array}$ & 0,00 & 0,02 & 0,30 & 0,13 & 0,01 & 0,08 \\
\hline $\begin{array}{l}\text { Faturamento } \\
\text { por cliente }\end{array}$ & 0,00 & 0,00 & 0,30 & 0,24 & 0,00 & 0,00 \\
\hline
\end{tabular}

Fonte: Pesquisa de campo.

Os resultados mostram que dois dos indicadores de desempenho referentes a clientes (satisfação e fidelidade) não apresentaram rejeição à hipótese nula em nenhuma das combinações analisadas, sugerindo que a intensidade de uso destes indicadores de desempenho pode ser considerada como independente da função 
operacional exercida pelas empresas individuais. As percentagens de utilização revelam que o indicador de desempenho referente à satisfação dos clientes tem sido amplamente utilizado por empresas que atuam em todas as funções testadas. Os resultados obtidos relativos aos demais indicadores de desempenho desta perspectiva que foram testados apresentam rejeições à nula hipótese, o que indica diferenças significativas nos padrões de adoção.

De maneira semelhante, os mesmos procedimentos estatísticos foram adotados acerca dos indicadores referentes aos processos internos. Os resultados estão apresentados na tabela 4.

Tabela 4. Resultados da prova Exata de Fisher acerca dos indicadores de processos internos.

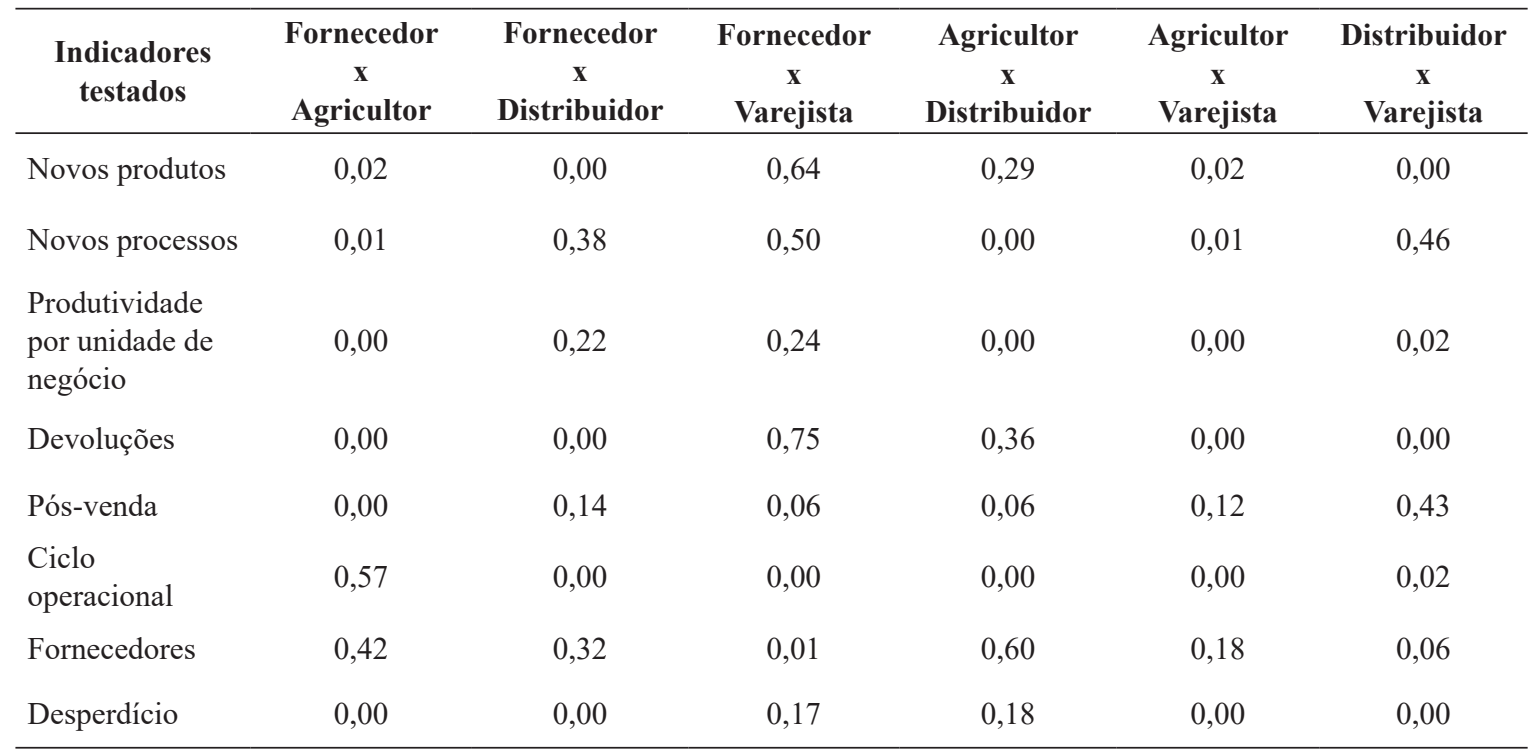

Fonte: Pesquisa de campo.

Apenas o indicador de desempenho relativo à resposta aos clientes não apresentou rejeição da hipótese nula. Este resultado indica que a intensidade de uso deste indicador pode ser considerada como similar entre as funções operacionais testadas. Por outro lado, indicadores de desempenho tradicionais de processos internos (pós venda, fornecedores, tempo de armazenamento, atraso na entrega, volume de negócios de produtos, ciclo operacional e flexibilidade) apresentaram rejeições à hipótese nula.

Finalmente, este teste foi realizado utilizando os indicadores de desempenho associados a aprendizado e crescimento. Os resultados são apresentados na tabela 5. Os resultados obtidos apontam que todos os indicadores testados mostram evidências estatisticamente significativas acerca de rejeições da hipótese nula, indicando a presença de diferenças significativas sobre a intensidade de uso entre as funções operacionais.

Tabela 5. Resultados da prova Exata de Fisher acerca dos indicadores de aprendizagem e crescimento.

\begin{tabular}{|c|c|c|c|c|c|c|}
\hline $\begin{array}{l}\text { Indicadores } \\
\text { testados }\end{array}$ & $\begin{array}{c}\text { Fornecedor } \\
\mathbf{x} \\
\text { Agricultor }\end{array}$ & $\begin{array}{c}\text { Fornecedor } \\
\mathbf{x} \\
\text { Distribuidor }\end{array}$ & $\begin{array}{c}\text { Fornecedor } \\
\mathbf{x} \\
\text { Varejista } \\
\end{array}$ & $\begin{array}{c}\text { Agricultor } \\
\mathbf{x} \\
\text { Distribuidor }\end{array}$ & $\begin{array}{c}\text { Agricultor } \\
\quad \mathbf{x} \\
\text { Varejista }\end{array}$ & $\begin{array}{c}\text { Distribuidor } \\
\mathbf{x} \\
\text { Varejista }\end{array}$ \\
\hline $\begin{array}{l}\text { Investimento em } \\
\text { treinamento }\end{array}$ & 0,00 & 0,00 & 0,00 & 0,06 & 0,07 & 0,58 \\
\hline $\begin{array}{l}\text { Investimento em } \\
\text { TI }\end{array}$ & 0,00 & 0,00 & 0,17 & 0,28 & 0,00 & 0,00 \\
\hline $\begin{array}{l}\text { Investimento em } \\
\text { SI }\end{array}$ & 0,00 & 0,00 & 0,50 & 0,06 & 0,00 & 0,02 \\
\hline Motivação & 0,32 & 0,50 & 0,00 & 0,36 & 0,07 & 0,00 \\
\hline $\begin{array}{l}\text { Capacidade dos } \\
\text { funcionários }\end{array}$ & 0,15 & 0,00 & 0,00 & 0,36 & 0,12 & 0,17 \\
\hline $\begin{array}{l}\text { Eficiência } \\
\text { gerencial }\end{array}$ & 0,00 & 0,01 & 0,50 & 0,07 & 0,00 & 0,02 \\
\hline $\begin{array}{l}\text { Satisfação dos } \\
\text { funcionários }\end{array}$ & 0,27 & 0,20 & 0,00 & 0,55 & 0,00 & 0,00 \\
\hline
\end{tabular}

Fonte: Pesquisa de campo. 
A partir dos resultados obtidos com a operacionalização da Prova Exata de Fisher, foram identificados 12 indicadores de desempenho que obtiveram quatro ou mais rejeições da hipótese nula testada. Estes indicadores foram utilizados na análise de agrupamentos Os resultados relativos ao grupo de empresas que atuam como fornecedores de insumos estão apresentados no gráfico 1.

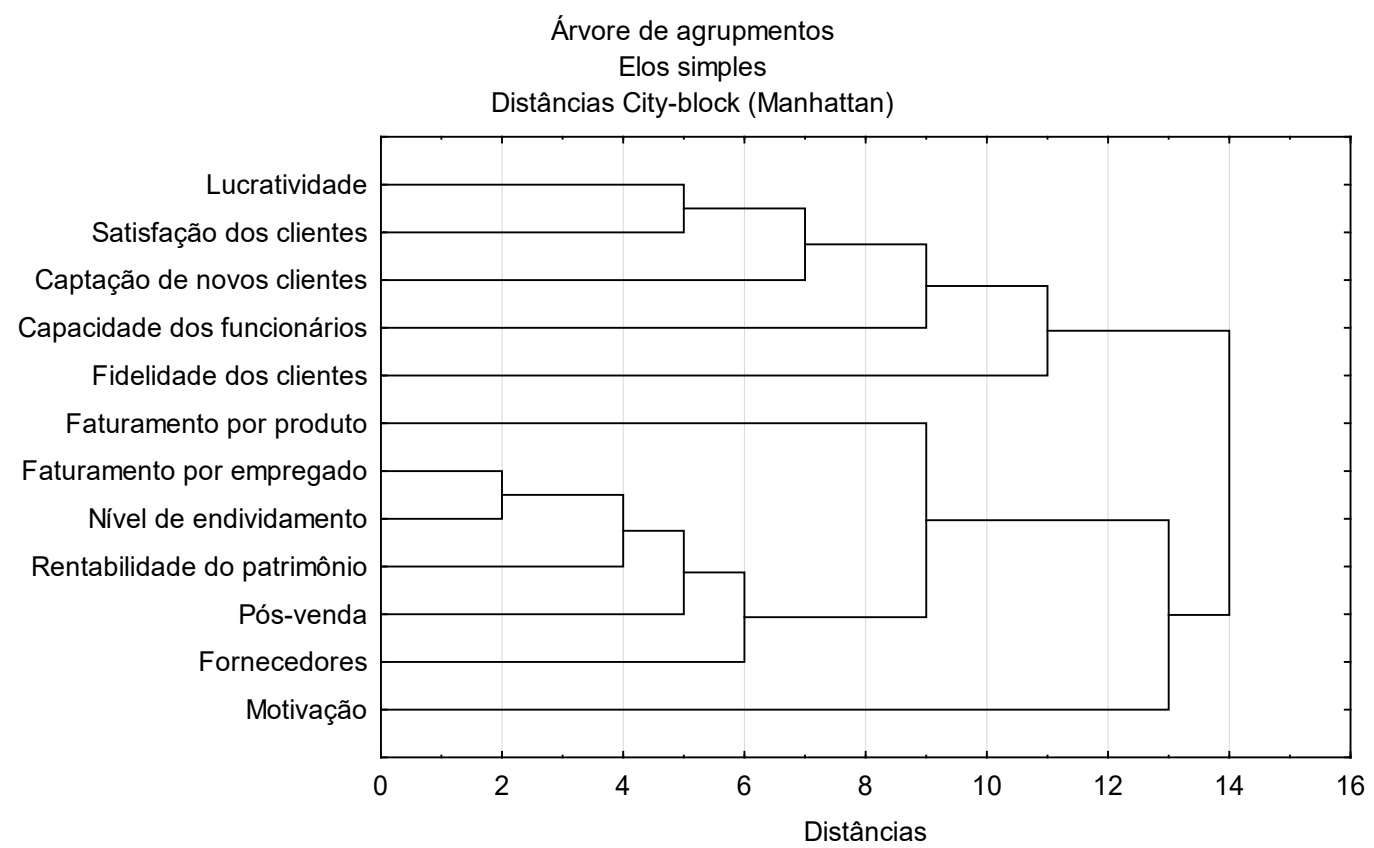

Gráfico 1. Distribuição gráfica dos indicadores através da árvore de agrupamentos (fornecedores de insumos) Fonte: Cálculos próprios.

A árvore de agrupamentos destaca a presença de dois grupos significativos. O primeiro grupo possui dois indicadores (lucratividade e satisfação dos clientes), enquanto o segundo é composto por três indicadores de desempenho (faturamento por empregado e nível de endividamento, bem como por rentabilidade do patrimônio de maneira adjacente). O primeiro grupo identificado é composto por indicadores que focam relações das empresas com seus clientes. O segundo grupo apresenta um perfil mais técnico voltado para a avaliação financeira das empresas. Estes resultados demonstram que as empresas que atuam nesta função operacional compartilham foco gerencial dual no que se refere aos aspectos relevantes de desempenho. A análise de agrupamentos também foi realizada entre os agricultores e os resultados estão dispostos no gráfico 2

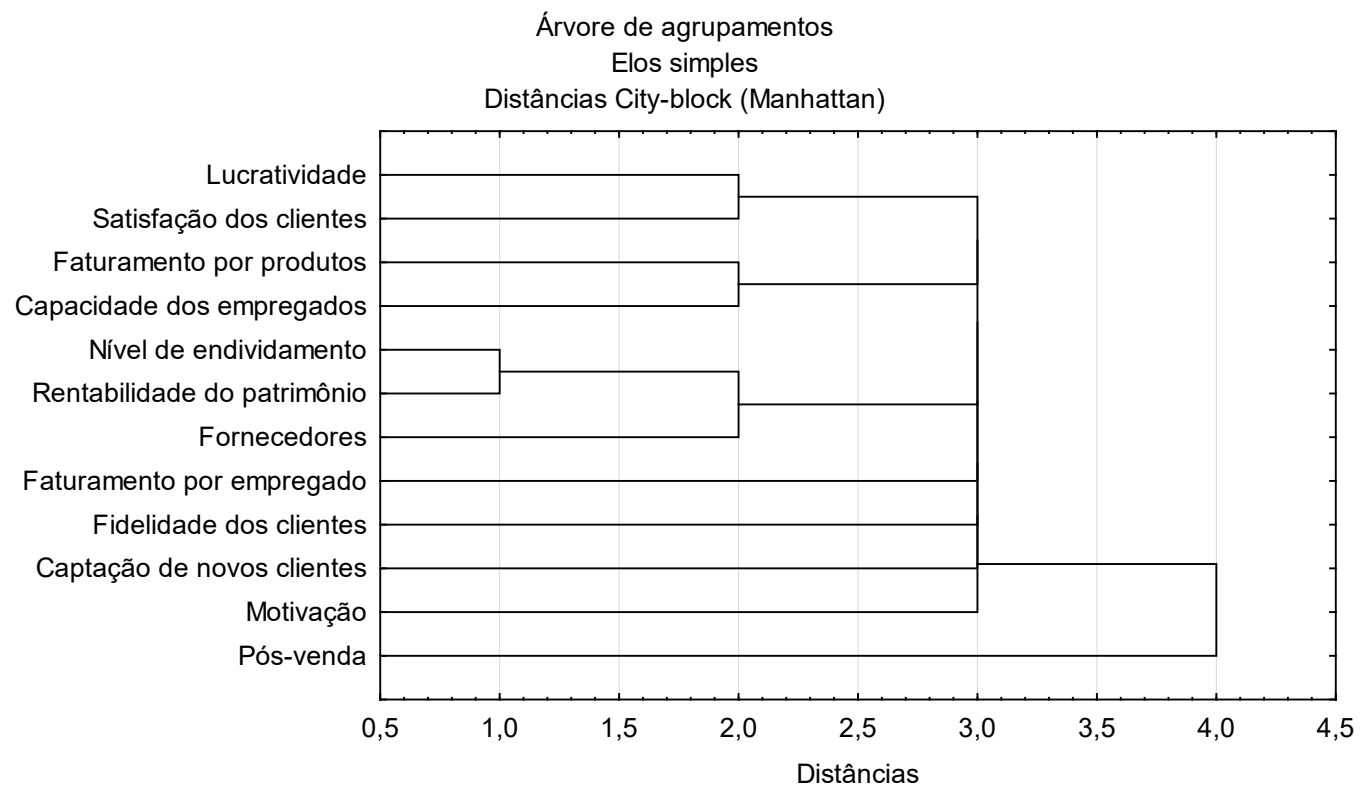

Gráfico 2. Distribuição gráfica dos indicadores através da árvore de agrupamentos (agricultores)

Fonte: Cálculos próprios. 
Para estas empresas individuais, a árvore de agrupamentos identificou a presença de três grupos significativos compostos. O primeiro grupo apresenta lucratividade e satisfação dos clientes, enquanto o segundo é composto pelos indicadores referentes ao faturamento por produto e a capacidade dos empregados. O terceiro grupo encontrado é composto por três indicadores (nível de endividamento, rentabilidade do patrimônio e fornecedores). Pode-se constatar que o primeiro dos três grupos encontrados é idêntico ao primeiro grupo identificado entre os fornecedores de insumos e que foi observada uma alteração na composição do núcleo principal do segundo grupo. Além disso, foi identificada uma vertente gerencial de desempenho adicional associado ao monitoramento do faturamento por produto e à capacidade dos funcionários. Este grupo apresenta um perfil eminentemente operacional. Vê-se que os resultados sugerem a presença de focos gerenciais específicos entre as empresas individuais que atuam nesta função operacional em comparação às empresas fornecedoras de insumos no que se refere aos aspectos relevantes de desempenho.

O mesmo procedimento foi operacionalizado para as empresas individuais que atuam como distribuidoras, chegando-se aos resultados apresentados no gráfico 3.

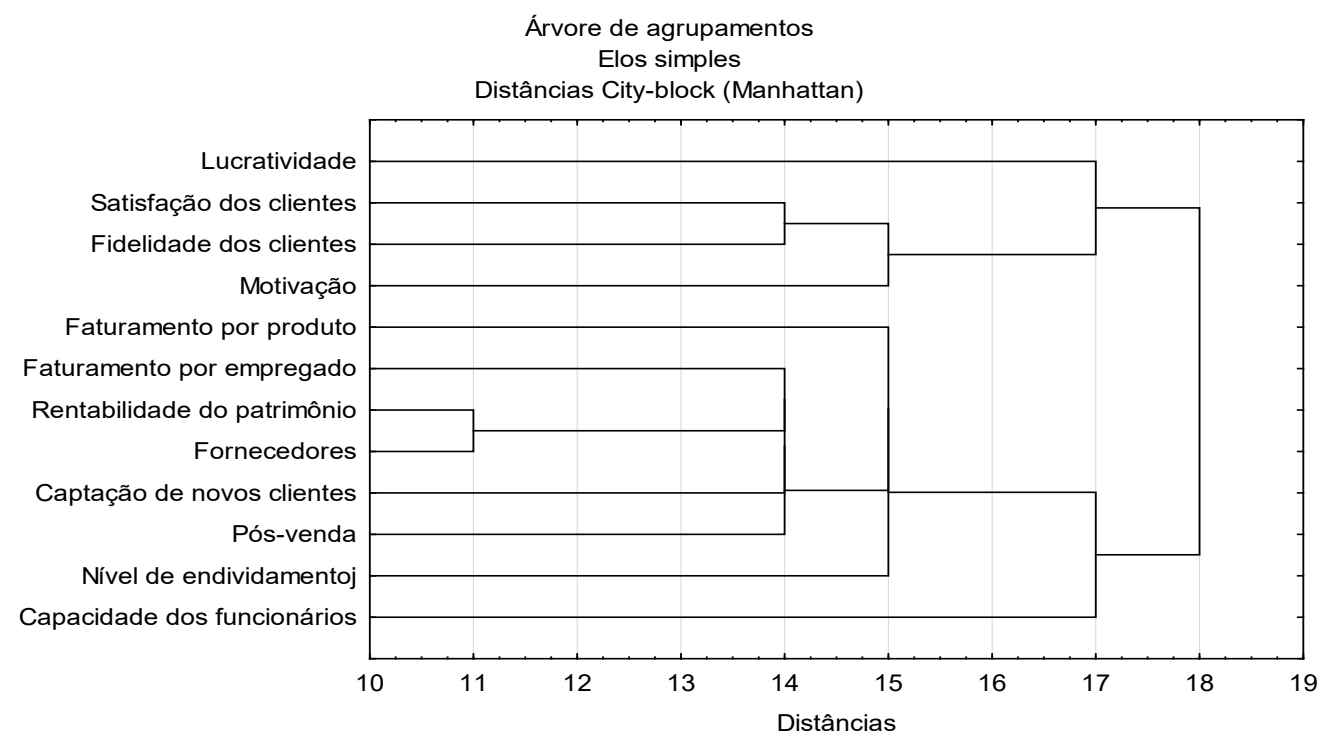

Gráfico 3. Distribuição gráfica dos indicadores através da árvore de agrupamentos (distribuidores)

Fonte: Cálculos próprios.

Para este grupo de empresas individuais, a árvore de agrupamentos destacou a presença de dois grupos significativos compostos por dois indicadores de desempenho. O primeiro grupo é composto pelos indicadores associados à satisfação e fidelidade dos clientes, enquanto o segundo é composto por cinco indicadores (faturamento por empregado, rentabilidade do patrimônio, fornecedores, captação de novos clientes e pósvenda). Pode-se observar que o primeiro grupo identificado possui um perfil exclusivamente focado nos clientes das empresas que atuam como distribuidoras, enquanto o segundo grupo possui foco gerencial na em aspectos financeiros e operacionais internos. Os resultados deixam clara a perspectiva específica acerca do foco gerencial sobre a mensuração de desempenho das empresas que possuem função operacional associada á distribuição em comparação aos resultados encontrados para fornecedores de insumos e agricultores.

Finalmente, a análise de agrupamentos também foi realizada entre os varejistas. Os resultados estão dispostos no gráfico 4. Para estas empresas investigadas que atuam como varejistas, a árvore de agrupamentos identificou a presença de três grupos significativos compostos por dois indicadores de desempenho cada. $\mathrm{O}$ primeiro grupo é composto pelos indicadores referentes à lucratividade e à satisfação dos clientes é possui idêntica formação a um dos grupos encontrados entre os agricultores. O segundo é composto pelos indicadores de desempenho acerca do faturamento por produto e da rentabilidade do patrimônio. O terceiro grupo é formado pelos indicadores referentes à motivação e capacidade dos funcionários. Este último possui um foco gerencial centrados nos empregados que atuam profissionalmente nestas empresas. 


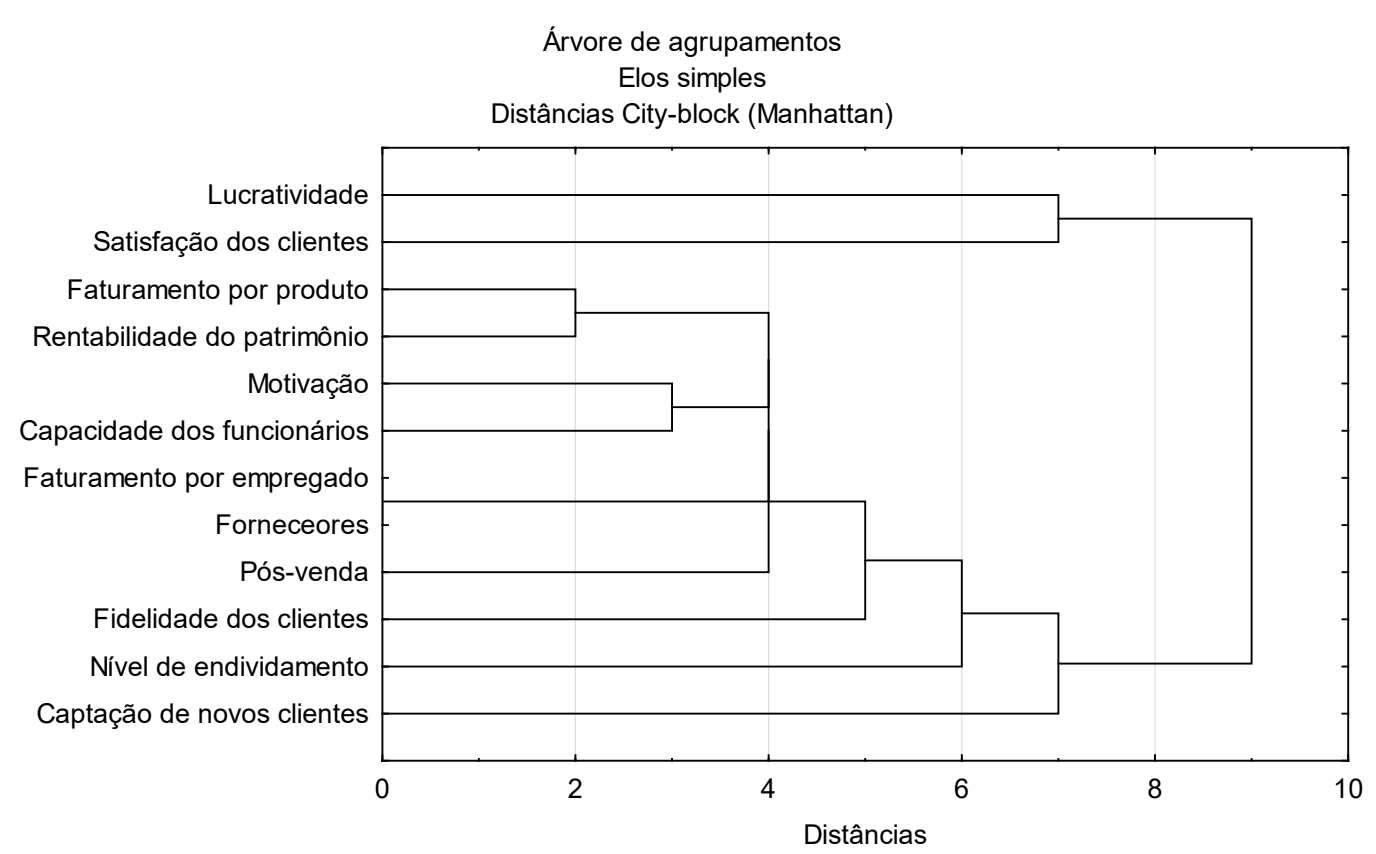

Gráfico 4. Distribuição gráfica dos indicadores através da árvore de agrupamentos (varejistas)

Fonte: Cálculos próprios.

Estes resultados corroboram presença de focos gerenciais associados à mensuração do desempenho específicos para cada um dos grupos de empresas investigadas a partir de suas respectivas funções operacionais.

\section{CONCLUSÕES}

O objetivo deste artigo foi identificar a presença de especificidades acerca da adoção de indicadores de desempenho a partir de uma abordagem institucional advinda de distintas funções operacionais executadas por empresas individuais no âmbito de uma cadeia agroalimentar. $\mathrm{Na}$ análise, ficou clara a relevância dos aspectos institucionais acerca da medição de desempenho no contexto de cadeias de suprimento considerando as especificidades das diversas empresas individuais que a compõem.

Os resultados fornecem evidências empíricas acerca de que especificidades gerenciais das empresas estejam relacionadas às funções operacionais que elas executam e suas respectivas posições na estrutura da cadeia de suprimento e suas rotinas operacionais possam influenciar a importância de distintos grupos de indicadores de desempenho, que podem receber maior ou menor importância, de acordo com sua contribuição operacional, indicando uma hierarquia de valor entre indicadores de desempenho.

Já a presença de distintas dimensões de medição de desempenho pôde ser vista, tendo sido representada pela identificação de métricas comuns e específicas, bem como pela presença de distintas importâncias relativas no contexto de cada uma das funções operacionais analisadas. Isso dá suporte à presença de especificidades de adoção de indicadores de desempenho, bem como sugere que certos indicadores de desempenho individual podem ser muito relevantes para funções específicas, mesmo que não obtenham padrões de uso similares entre as diversas funções. A viabilidade de um sistema de mensuração de desempenho de cadeia de suprimento que considere tanto indicadores comuns e específicos ainda não está clara.

Novos estudos poderão gerar informações mais detalhadas sobre a natureza das relações dinâmicas entre esses dois aspectos e, assim, contribuir para a melhoria do conhecimento existente sobre este assunto. 


\section{REFERÊNCIAS}

AKYUZ, G.A.; ERKAN, T.E. Supply chain performance measurement: a literature review. International Journal of Production Research, v. 48, n. 17, pp. 5137-5155, 2010.

BEAMON, B. Supply chain and analysis models and methods. International Journal of Production Economics, v. 55, n. 3, pp. 281-294, 1998.

Measuring supply chain performance. International Journal of Operations and Production Management, v. 19, n. 3, pp. 275-292, 1999.

BHAGWAT, R.; SHARMA, M.K. Performance measurement of supply chain management: a balanced scorecard approach. Computer \& Industrial Engineering, v. 53, n. 1, pp. 43-62, 2007.

BIGLIARDI, B.; BOTTANI, E. Performance measurement in the food supply chain: a balanced scorecard approach. Facilities, v. 28, n. 5/6, pp. 249-260, 2010.

BRAZILIAN FRUIT. Fruticultura. Disponível em at http://brazilianfruit.org.br/ing/fruticultura/fruticultura.asp. Acesso em: 23 set 2014.

BREWER, P.C.; SPEH, T.W. Using the balanced scorecard to measure supply chain performance. Journal of Business Logistics, v. 21, n. 1, pp. 75-93, 2000.

CABRAL, R,M. Custos de transação. In: CALlADO, A.A.C. (Org). Agronegócio. 3ed. São Paulo: Atlas, 2011. Pp. 105-119.

CAlladO, A.A.C.; MENDES, E.; CALlADO, A.L.C. Um estudo empírico da significância das relações entre a elaboração de metas estratégicas e o uso de indicadores de desempenho. Revista Iberoamericana de Contabilidad de Gestión, v. 11, n. 21, p. 1-15, 2013

CAlladO, A.A.C.; PINHO, M.A.B. de. Evidências de Isomorfismo Mimético sobre Práticas de Gestão de Custos entre Micro e Pequenas Empresas de Diferentes Setores de Atividade. Contabilidade Vista \& Revista, v. 25, n. 2, p. 1119-137, 2014.

CARVALHO, C.A.P.; VIEIRA, M.M.; LOPES, F.D. Perspectiva Institucional para análise das organizações. In: Encontro Anual da Associação Nacional dos Programas de Pós-Graduação em Administração 23., 1999, Rio de Janeiro. Anais... Rio de Janeiro/RJ:ANPAD, 1999. CD-ROM.

CHAN, F.T.S.; QI, H.J. An innovative performance measurement method for supply chain management. Supply Chain Management: An International Journal, v. 8, n. 3, pp. 209-223, 2003.

CHAN, F.T.S.; QI, H.J.; CHAN, H.K.; LAU, H.C.W.; IP, R.W.L. A conceptual model of performance measurement for supply chains. Management Decision, v. 41, n. 7, pp. 635-642, 2003.

CHANG, H.H. An empirical study of evaluating supply chain management integration using the balanced scorecard in Taiwan. The Service Industries Journal, v. 29, n. 2, pp. 185-202, 2009.

CHENHALL, R.H. Integrative strategic performance measurement systems, strategic alignment of manufacturing, learning and strategic outcomes: an exploratory study. Accounting, Organization and Society, v. 30, n. 5, pp. 395-422, 2005.

CHIA, A. GOH, M. HUM, S. Performance measurement in supply chain entities: balanced scorecard perspective. Benchmarking: An International Journal, v. 16, n. 5, pp. 605-620, 2009.

CHRISTOPHER, M. Logistics and Supply Chain Management. London, Prentice Hall, 2005.

DiMAGGIO, P.; POWELL, W. The Iron Cage Revisited: Institutional Isomorphism and Collective Rationality in Organizational Fields. In: POWELL, W., DiMAGGIO, P. The New Institutionalism in Organizational Analysis. Chicago: University of Chicago Press, 1991.

DUBOIS, A.; HUKTHÉN, K.; PEDERSEN, A-C. Supply chains and interdependence: a theoretical analysis. Journal of Purchasing \& Supply Management, v. 10, n. 1, pp. 3-9, 2004.

ELROD, C.; MURRAY, C.; BANDE, S. A review of performance metrics for supply chain management. Engineering Management Journal, v. 25, n. 3, pp. 39-50, 2013.

FAO - Food and Agriculture Organization. FAO Statistical yearbook. World Food and Agriculture. Disponível 
em http://issuu.com/faooftheun/docs/syb2013issuu/156. Acesso em: 25 set 2014.

FOLAN, P.; BROWNE, J. A review of performance measurement: Towards performance management. Computers in Industry, v. 56, n. 7, pp. 663-680, 2005

HENSON, S.; REARDON, T. Private agri-food standards: Implications for food policy and the agri-food system. Food Policy. v. 30, p. 241-253, 2005.

IBGE - Instituto Brasileiro de Geografia e Estatística. Censo Agropecuário 2006. Disponível em http://www.ibge. gov.br/home/estatistica/economia/agropecuaria/censoagro/default.shtm. Acesso em: 12 fev 2013.

GANGA, G.M.D.; CARPINETTI, L.C.R. A fuzzy logic approach to supply chain performance management. International Journal of Production Economics, v. 134, n. , pp. 177-187, 2011.

GUNASEKARAN, A; PATEL, C., MCGAUGHEY, R.E. A framework for supply chain performance measurement. International Journal of Production Economics, v.87, n. 3, pp. , 2004.

HAAN, J. DE.; GROOT, G. DE.; LOO, E. YPENBURG, M. Flows of goods or supply chains: lessons from the natural rubber industry in Kerala, India. International Journal of Production Economics, v. 81-82, n. 11, pp. 185-194, 2003.

HAIR, J. F.; ANDERSON, R. E.; TATHAM, R. L.; BLACK, W. C. Análise Multivariada de Dados. Porto Alegre: Bookman, 2005.

HENSON, S., REARDON, T. Private agri-food standards: Implications for food policy and the agri-food system. Food Policy, v. 30, n. 3, pp. 241-253, 2005.

HOFMAN, D. The hierarchy of supply chain metrics. Supply Chain Management Review, v. 8, n. 6, pp. 28-37, 2004.

HOLMBERG, S. A systems perspective on supply chain measurements. International Journal of Physical Distribution and Logistics, v. 30, n. 10, pp. 847-868, 2000.

KAPLAN, Robert S; COOPER, Robin. Custo \& desempenho: administre seus custos para ser mais competitivo. São Paulo: Futura, 1998.

KOLEHMAINEN, K. Dynamic strategic performance measurement systems: balancing empowerment and alignment. Long Range Planning, v. 43, n. 4, pp. 527-554, 2010.

LAMBERT, D.M.; POHLEN, T.L. Supply chain metrics. The International Journal of Logistics Management, v. 12, n. 1, pp. 1-19, 2001.

LEE, C.W.; KWON, I.G.; SEVERANCE, D. Relationship between supply chain performance and degree of linkage among supplier, internal integration, and customer. Supply Chain Management: An International Journal. V. 12, n. 6, pp. 444-452, 2007.

LEVIN, J. Estatística aplicada a ciências humanas. 2. ed. São Paulo: Harbra, 1987.

LOHMAN, C.; FORTUIN, L.; WOUTERS, M. Designing a performance measurement system: a case study. European Journal of Operational Research, v. 156, n. 2, pp. 267-286, 2004.

MAPA - Ministério da Agricultura, Pecuária e Abastecimento. Cadeia produtiva de frutas. Buainain, A.M., and Batalha, M.O. (Org.), Série Agronegócios, 7, Disponível em http://www.ibraf.org.br/x files/Documentos/ Cadeia Produtiva de Frutas_S\%C3\%A9rie Agroneg\%C3\%B3cios MAPA.pdf. Aceso em: 23 dez 2014.

MATOPOULOS, A.; VLACHOPOULOU, M.; MANTHOU, V. A conceptual framework for supply chain collaboration: empirical evidence from the agri-food industry. Supply Chain Management: An International Journal, v. 12, n. 3, pp. 177-186, 2007.

MELNYK, S.A.; STEWART, D.M.; SWINK, M. Metrics and performance measurement in operations management: dealing with the metrics maze. Journal of Operations Management, v. 22, n. 3, pp. 209-217, 2004.

MEYER, J. W., SCOTT, W. R., DEAL, T. E. Institutional and technical sources of organizational structure. In: MEYER, J. W., SCOTT, W. R. Organizational Environments: Ritual and Rationality. Newbury Park: Sage, 1992.

MIN, S.; MENTZER, J.T.; LADD, R.T. A market orientation in supply chain management. Journal of the Academy of Market Sciences, v. 35, n. 4, pp. 507-522, 2007. 
MORASH, E.A. Supply chain strategies, capabilities and performance. Transportation Journal, v. 41, n. 11, pp. 37-54, 2002.

NEELY, A.; GREGORY, M.; PLATTS, K. Performance Measurement System Design: a literature review and research agenda. International Journal of Operations \& Production Management, v. 15, n. 4, pp. 80-116, 1995.

NEVES, M.F. Canais de distribuição no agronegócio: conceitos básicos. In: NEVES, M.F.; CASTRO, L.T. (Org.) Marketing e estratégia em agronegócios e alimentos. São Paulo: Atlas, 2003. pp. 223-249.

PARK, J.H.; LEE, J.K.; YOO, J.S. A framework for designing the balanced supply chain scorecard. European Journal of Information Systems, v. 14, pp. 335-346, 2005.

RAJESH, R.; PUGAZHENDHI, S.; GANESH, K.; DUCQ, Y.; KOH, S.C.L. Generic balanced scorecard framework for third party logistics service provider. International Journal of Production Economics, v. 140, n. 1, pp. 269-282, 2012.

SELZNICK, P. Institutionalism “old” and "new”. Administrative Science Quarterly, Ithaca, v. 41, n.2, pp.270277, 1996.

VAN DER VORST, J.G.A.J. Performance measurement in agri-food supply chain networks. In: ONDERSTEIJN, C.M.D.; WIJNANDS, J.H.M.; HUIRNE, B.M.; VAN KOOTEN (Coord.), O. Quantifying the agri-food supply chain. Spring, 2006.

VAN HOEK, R.I. Measuring the unmeasurable - measuring and improving performance in the supply chain. Supply Chain Management, v. 3, n. 4, pp. 187-192, 1998.

. The contribution of performance measurement to the expansion of third party logistics alliances in the supply chain. International Journal of Operations \& Production Management, v. 21, n. 1/2, pp. 15-29, 2001.

VARMA, S.; WADHWA, S.; DESHMUKH, S.G. Evaluating petroleum supply chain performance: application of analytical hierarchy process to balanced scorecard. Asian Pacific Journal of Marketing and Logistics, v. 20, n. 3, pp. 343-356, 2008.

WU, I.; CHANG, C. Using the balanced scorecard in assessing the performance of e-SCM diffusion: a multi-stage perspective. Decisions Support Systems, v. 52, n. 2, pp. 474-485, 2012.

WILLIAMSON, O.E. Markets and Hierarchies: Analysis and Antitrust Implications. New York: Free Press, 1975.

ZUCKER, L.G. Institutional Theories of Organization. Annual Review of Sociology, v. 13, pp. 443-464, 1987. 